\title{
Association of total bilirubin and indirect bilirubin content with metabolic syndrome among Kazakhs in Xinjiang
}

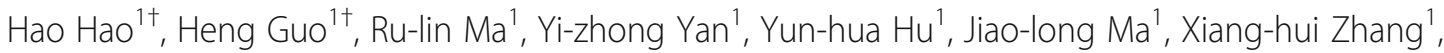
Xin-ping Wang ${ }^{1}$, Kui Wang ${ }^{1}$, La-ti Mu' ${ }^{1}$, Yan-peng Song ${ }^{1,2}$, Jing-yu Zhang ${ }^{1}$, Jia He ${ }^{1 *}$ and Shu-xia Guo ${ }^{1,3^{*}}$

\begin{abstract}
Background: Some studies have shown that a high level of bilirubin is a protective factor against metabolic syndrome (MS), while a high level of transaminase is a risk factor for MS. However, the existing results are inconsistent and few cohort studies have been published.

Methods: Using an ambispective cohort study, 565 Kazakhs from Xinjiang, China were selected as the study subjects. The baseline serum bilirubin and transaminase levels of the subjects were divided into quartiles and the relationship between these values and the incidence of MS was analyzed. The definition of MS was based on the Joint Interim Statement (JIS) diagnostic criteria.

Results: The average follow-up time for the subjects was 5.72 years. The cumulative incidence of MS was $36.11 \%$ (204 of the 565 subjects), and the incidence density was 63.10/1000 person-years. Multivariate Cox regression analysis showed that the levels of total bilirubin (TBIL) and indirect bilirubin (IBIL) were negatively correlated with the occurrence of MS, Compared to the lowest quartile level (Q1), the hazard ratios of MS the TBIL levels at the Q2Q4 quartiles were: $0.47(0.31-0.71), 0.53(0.35-0.79)$, and $0.48(0.32-0.72)$, respectively, while IBIL levels at the Q2-Q4 quartiles showed an MS hazard ratio of $0.48(0.32-0.72), 0.54(0.36-0.81)$, and $0.52(0.35-0.77)$, respectively, all at a 95\% confidence level. However, no relationship was found between transaminase levels and the incidence of MS.

Conclusion: Serum TBIL and IBIL levels were negatively correlated with the incidence of MS in a Kazakh population in China.
\end{abstract}

Keywords: Bilirubin, Transaminase, Metabolic syndrome

*Correspondence: hejia123.shihezi@163.com; gsxshzu@sina.com

${ }^{\dagger} \mathrm{Hao}$ Hao and Heng Guo contributed equally to this work.

${ }^{1}$ Department of Public Health, Shihezi University School of Medicine, Shihezi

832000, Xinjiang, China

Full list of author information is available at the end of the article

(C) The Author(s). 2020 Open Access This article is licensed under a Creative Commons Attribution 4.0 International License, which permits use, sharing, adaptation, distribution and reproduction in any medium or format, as long as you give appropriate credit to the original author(s) and the source, provide a link to the Creative Commons licence, and indicate if changes were made. The images or other third party material in this article are included in the article's Creative Commons licence, unless indicated otherwise in a credit line to the material. If material is not included in the article's Creative Commons licence and your intended use is not permitted by statutory regulation or exceeds the permitted use, you will need to obtain permission directly from the copyright holder. To view a copy of this licence, visit http://creativecommons.org/licenses/by/4.0/ The Creative Commons Public Domain Dedication waiver (http://creativecommons.org/publicdomain/zero/1.0/) applies to the data made available in this article, unless otherwise stated in a credit line to the data. 


\section{Background}

Metabolic syndrome (MS) is a clustering of complex medical conditions, including hypertension, hyperglycemia, central obesity, hypertriglyceridemia, and high-density lipoprotein cholesterol (HDL-C) reduction. MS can lead to cardiovascular disease, type 2 diabetes, lipid disorder, liver steatosis, and other circulatory system disorders [1]. Although the etiology and pathogenesis of MS are not fully understood, it is generally believed that factors such as insulin resistance, inflammation, and oxidative stress play an important role in its occurrence and development [1-3]. Bilirubin, the main metabolite of heme, is toxic, and can cause irreversible damage to the brain and nervous system. Liver cells remove bilirubin from the body through its intake, transformation, and excretion. High levels of bilirubin in the serum indicate liver dysfunction. However, increasing evidence suggests that serum bilirubin has anti-inflammatory and antioxidant functions, and can also improve vascular endothelial function and enhance insulin sensitivity $[4,5]$, suggesting that a high level of bilirubin can protect against MS [6,7]. Transaminase can promote inflammation and induce insulin resistance [8] and studies have shown that a high level of the transaminase alanine aminotransferase (ALT) is a risk factor for MS $[6,9]$. One cohort study showed that $\gamma$-glutamyl transpeptidase levels in men and ALT levels in women are related to MS, but that direct bilirubin (DBIL), total bilirubin (TBIL), ALT, and aspartate aminotransferase (AST) levels in men and DBIL, TBIL, and AST levels in women are not [10]. However, further research is clearly needed on this topic, as the results of the previous studies are inconsistent.

Kazakhs in Xinjiang, China are nomadic minorities living primarily in remote mountainous areas. The majority fall into a low economic class and their culture and customs are significantly different from those of other ethnic groups $[11,12]$. A previous study by our research group found the prevalence of MS in Kazakhs to be higher than that in other ethnic groups in Xinjiang and higher than the national average [13]. Therefore, this study used Xinjiang Kazakh residents as the research group and explored the relationship between the incidence of MS and serum bilirubin and transaminase levels. The results of this study provide potential advances for the early diagnosis and prevention of MS in the Xinjiang Kazakh population; our results may be extended to people living in Kazakhstan and Uzbekistan as well.

\section{Methods}

\section{Research subjects}

Based on the geographical distribution of Xinjiang's ethnic minorities in northwestern China, a representative county (six villages in the Nalati Township, Xinyuan
County) was selected and an ambispective cohort study was conducted on the local Kazakh population. The study baseline was conducted in 2009 and 2012 and a follow-up was performed in 2013, 2016, and 2017. The study included 785 Kazakh permanent residents aged 18 and over who voluntarily participated in the survey; 10 subjects with chronic liver disease, 2 subjects with cancer, and 208 subjects with MS at baseline were excluded from the study, resulting in a total of 565 subjects.

\section{Epidemiological investigation and biochemical tests}

At baseline and at each follow-up, a face-to-face interview was conducted by uniformly trained investigators to collect personal information, disease history, family history, and lifestyle behavior. Height, weight, waist circumference (WC), systolic blood pressure (SBP), and diastolic blood pressure (DBP) were measured and recorded according to standardized methods. TBIL, DBIL, indirect bilirubin (IBIL), ALT, AST, fasting plasma glucose (FPG), triglyceride (TG), total cholesterol (TC), HDL-C, and low-density lipoprotein cholesterol (LDL-C) levels were measured using an automatic biochemical analyzer (Olympus Au 2700; Olympus diagnostics, Hamburg, Germany). Each subject signed an informed consent form. The investigation was approved by the ethics review committee of the First Affiliated Hospital of Shihezi University Medical College (IERB No. SHZ2010LL01), and the study methods were carried out in accordance with the relevant guidelines.

\section{Definition of MS}

Our identification of MS is based on the Joint Interim Statement (JIS) diagnostic criteria [14]. For MS to be diagnosed, three or more of the following indicators must be observed: (1) men $\mathrm{WC} \geq 85 \mathrm{~cm}$, women $\mathrm{WC} \geq 80 \mathrm{~cm}$; (2) $\mathrm{SBP} \geq 130 \mathrm{mmHg}$ or $\mathrm{DBP} \geq 85 \mathrm{mmHg}$, have received treatment for hypertension, or have been previously diagnosed with hypertension; (3) $\mathrm{FPG} \geq 5.6 \mathrm{mmol} / \mathrm{L}$, have received treatment for type 2 diabetes, or have been previously diagnosed with type 2 diabetes. (4) TG $\geq 1.70$ $\mathrm{mmol} / \mathrm{L}$ or received treatment for elevated triglycerides; (5) men $\mathrm{HDL}-\mathrm{C}<1.0 \mathrm{mmol} / \mathrm{L}$, women $\mathrm{HDL}-\mathrm{C}<1.30$ $\mathrm{mmol} / \mathrm{L}$.

\section{Statistical analysis}

Data analysis was performed using SPSS version 20.0 (Chicago, Illinois, USA). Means \pm standard deviations were used to describe continuous variables, and percentages were used to describe categorical variables. Rank sum tests were used to compare baseline characteristics between men and women, and chi-square tests were used to compare categorical variables. Serum bilirubin and transaminase levels in this population differed between men and women. Therefore, the baseline levels 
were separated into quartiles (Q1, Q2, Q3, and Q4) for each gender. The multivariate Cox proportional-hazards model was used to analyze the relationship between serum bilirubin or transaminase levels and MS. After adjusting for age and gender, the hazard ratio (HR) and 95\% confidence interval (95\% CI) of serum bilirubin and transaminase levels to MS were calculated. All statistical tests were bilateral and $P<0.05$ indicated statistical significance.

\section{Results}

\section{Baseline characteristics}

A total of 565 subjects were included in this survey, including 221 males (39.1\%). The average age of the population was $40.33 \pm 11.86$ years; the average age of men was significantly higher than that of women $($ men $=$ $42.10 \pm 12.93$ years; $\quad$ women $=39.20 \pm 10.99$ years; $\quad P=$ 0.011). In a comparison of baseline characteristics between the two genders, the levels of WC, SBP, DBP, TG, TBIL, IBIL, ALT, and AST were higher in men than women, and HDL-C levels were higher in women than in men. These differences were statistically significant $(P<0.05)$. These data are shown in Table 1.

\section{Incidence of MS}

Follow-up was conducted with the subjects for 3233.04 person-years, with an average follow up time of $5.72 \pm$
1.49 years per subject. During this period, a total of 204 subjects developed MS, with a cumulative incidence of $36.11 \%(95 \% \mathrm{CI}=0.32-0.40)$ and an incidence density of $63.10 / 1000$ person-years. Of the 221 men in the study, 70 developed MS, with a cumulative incidence of $31.67 \%$ (95\% CI $=0.26-0.38)$ and an incidence density of 55.71/ 1000 person-years. The cumulative incidence increased with age $\left(x^{2}\right.$ trend $\left.=43.166, P<0.001\right)$. Of the 344 women in this study, 134 developed MS, with a cumulative incidence of $38.95 \%(95 \% \mathrm{CI}=0.34-0.44)$ and an incidence density of $67.80 / 1000$ person-years. The cumulative incidence again increased with age $\left(x^{2}\right.$ trend $=76.391, P<0.001)$. There was no statistically significant difference in the cumulative incidence and incidence density between men and women. These data are shown in Table 2.

\section{Analysis of factors influencing MS}

We divided the levels of serum bilirubin and transaminase into quartiles and examined the relationship between the different quartile levels and the risk of MS. There was no significant difference in the cumulative incidence of MS at different quartile levels. Using serum bilirubin and transaminase levels as independent variables and the occurrence of MS as the dependent variable for Cox proportional hazards model analysis and adjusting for age and gender, we found that TBIL and IBIL levels were

Table 1 Baseline characteristics based on different genders

\begin{tabular}{|c|c|c|c|c|}
\hline & Total & Men & Women & $P$ \\
\hline Number (\%) & 565 & $221(39.1)$ & $344(60.9)$ & \\
\hline Age (years) & $40.33 \pm 11.86$ & $42.10 \pm 12.93$ & $39.20 \pm 10.99$ & 0.011 \\
\hline Smoking, N (\%) & $187(33.1)$ & 117 (52.9) & $70(20.3)$ & $<0.001$ \\
\hline Drinking, N (\%) & $55(9.7)$ & $52(23.5)$ & $3(0.9)$ & $<0.001$ \\
\hline WC (cm) & $81.58 \pm 9.97$ & $83.64 \pm 9.77$ & $80.26 \pm 9.88$ & $<0.001$ \\
\hline $\mathrm{SBP}(\mathrm{mmHg})$ & $127.84 \pm 21.72$ & $132.10 \pm 21.64$ & $125.11 \pm 21.36$ & $<0.001$ \\
\hline $\mathrm{DBP}(\mathrm{mmHg})$ & $81.92 \pm 13.79$ & $84.06 \pm 13.52$ & $80.54 \pm 13.81$ & $<0.001$ \\
\hline FPG (mmol/L) & $4.37 \pm 0.97$ & $4.48 \pm 1.12$ & $4.29 \pm 0.86$ & 0.214 \\
\hline $\mathrm{TG}(\mathrm{mmol} / \mathrm{L})$ & $1.05 \pm 0.84$ & $1.05 \pm 0.52$ & $1.05 \pm 0.99$ & 0.018 \\
\hline TC (mmol/L) & $4.13 \pm 0.94$ & $4.17 \pm 0.86$ & $4.11 \pm 0.99$ & 0.212 \\
\hline $\mathrm{HDL}-\mathrm{C}(\mathrm{mmol} / \mathrm{L})$ & $1.42 \pm 0.37$ & $1.36 \pm 0.36$ & $1.46 \pm 0.38$ & $<0.001$ \\
\hline LDL-C (mmol/L) & $2.08 \pm 0.67$ & $2.13 \pm 0.64$ & $2.04 \pm 0.69$ & 0.054 \\
\hline $\mathrm{BMI}\left(\mathrm{kg} / \mathrm{m}^{2}\right)$ & $23.26 \pm 3.44$ & $23.43 \pm 3.32$ & $23.15 \pm 3.51$ & 0.359 \\
\hline TBIL $(\mu \mathrm{mol} / \mathrm{L})$ & $10.84 \pm 4.70$ & $11.84 \pm 4.71$ & $10.19 \pm 4.59$ & $<0.001$ \\
\hline IBIL $(\mu \mathrm{mol} / \mathrm{L})$ & $8.08 \pm 3.82$ & $8.76 \pm 3.88$ & $7.65 \pm 3.73$ & $<0.001$ \\
\hline $\mathrm{DBIL}(\mu \mathrm{mol} / \mathrm{L})$ & $2.58 \pm 1.48$ & $2.72 \pm 1.56$ & $2.48 \pm 1.42$ & 0.054 \\
\hline ALT (IU/L) & $15.34 \pm 10.36$ & $16.43 \pm 8.28$ & $14.64 \pm 11.45$ & $<0.001$ \\
\hline AST (IU/L) & $27.37 \pm 12.48$ & $28.70 \pm 11.84$ & $26.52 \pm 12.82$ & 0.002 \\
\hline
\end{tabular}

WC waist circumference; SBP systolic blood pressure; DBP diastolic blood pressure; FPG fasting plasma glucose; TG Triglycerides; TC total cholesterol, $H D L-C$ highdensity lipoprotein cholesterol, $L D L-C$ low-density lipoprotein cholesterol; $B M I$ body mass index; $T B I L$ total bilirubin; $I B I L$ indirect bilirubin; $D B I L$ direct bilirubin, $A L T$ alanine aminotransferase; AST aspartate aminotransferase 
Table 2 Comparison of the incidence of metabolic syndrome in different age groups

\begin{tabular}{|c|c|c|c|c|c|c|}
\hline Group & Number & Number of MS & Cumulative incidence (\%) & Incidence density $\left(10^{-3}\right.$ person-years) & $X_{\text {trend }}^{2}$ & $P$ \\
\hline total & 565 & 204 & 36.11 & 63.10 & & \\
\hline men & 221 & 70 & 31.67 & 55.71 & & \\
\hline$<35$ & 69 & 14 & 20.29 & 36.75 & 43.166 & $<0.001$ \\
\hline $35-45$ & 70 & 23 & 32.86 & 54.96 & & \\
\hline$>45$ & 82 & 33 & 40.24 & 72.19 & & \\
\hline women & 344 & 134 & 38.95 & 67.80 & & \\
\hline$<35$ & 128 & 38 & 29.69 & 51.49 & 76.391 & $<0.001$ \\
\hline $35-45$ & 121 & 51 & 42.15 & 72.24 & & \\
\hline$>45$ & 95 & 45 & 47.37 & 84.52 & & \\
\hline
\end{tabular}

negatively correlated with MS, but there was no correlation between the DBIL, ALT, or AST levels and MS (Table 3). Compared to the lowest quartile level (Q1), the HRs $(95 \% \mathrm{CI})$ of TBIL Q2-Q4 levels were 0.47 (0.31-0.71), 0.53 (0.35-0.79), and 0.48 (0.32-0.72),

Table 3 Analysis of influencing factors for incident metabolic syndrome

\begin{tabular}{|c|c|c|c|c|c|}
\hline Parameter & $\mathrm{n}$ & MS,n(\%) & $H R$ & $95 \% \mathrm{Cl}$ & P \\
\hline \multicolumn{6}{|l|}{ TBIL } \\
\hline Q1 & 139 & $46(33.1)$ & ref & ref & ref \\
\hline Q2 & 141 & $50(35.5)$ & 0.47 & $0.31-0.71$ & $<0.001$ \\
\hline Q3 & 143 & $53(37.1)$ & 0.53 & $0.35-0.79$ & 0.002 \\
\hline Q4 & 142 & 55 (38.7) & 0.48 & $0.32-0.72$ & $<0.001$ \\
\hline \multicolumn{6}{|l|}{$\mid \mathrm{BI}$} \\
\hline Q1 & 140 & 46 (32.9) & ref & ref & ref \\
\hline Q2 & 141 & $48(34.0)$ & 0.48 & $0.32-0.72$ & $<0.001$ \\
\hline Q3 & 139 & $53(38.1)$ & 0.54 & $0.36-0.81$ & 0.003 \\
\hline Q4 & 145 & 57 (39.3) & 0.52 & $0.35-0.77$ & 0.001 \\
\hline \multicolumn{6}{|l|}{ DBIL } \\
\hline Q1 & 133 & $43(32.3)$ & ref & ref & ref \\
\hline Q2 & 135 & $54(40.0)$ & 1.23 & $0.82-1.84$ & 0.320 \\
\hline Q3 & 150 & $56(37.3)$ & 1.32 & $0.88-1.96$ & 0.178 \\
\hline Q4 & 147 & $51(34.7)$ & 0.88 & $0.58-1.32$ & 0.531 \\
\hline \multicolumn{6}{|l|}{ ALT } \\
\hline Q1 & 115 & $33(28.7)$ & ref & ref & ref \\
\hline Q2 & 194 & 68 (35.1) & 0.99 & $0.65-1.50$ & 0.949 \\
\hline Q3 & 131 & $55(42.0)$ & 1.06 & $0.69-1.63$ & 0.806 \\
\hline Q4 & 125 & 48 (38.4) & 1.04 & $0.67-1.62$ & 0.857 \\
\hline \multicolumn{6}{|l|}{ AST } \\
\hline Q1 & 130 & $44(33.8)$ & ref & ref & ref \\
\hline Q2 & 159 & $69(43.4)$ & 0.76 & $0.52-1.12$ & 0.169 \\
\hline Q3 & 136 & 49 (36.0) & 0.70 & $0.47-1.06$ & 0.091 \\
\hline Q4 & 140 & $42(30.0)$ & 0.86 & $0.56-1.31$ & 0.475 \\
\hline
\end{tabular}

Analysis adjusted for age, sex respectively, and those of the IBIL Q2-Q4 levels were 0.48 (0.32-0.72), 0.54(0.36-0.81), and 0.52 (0.35-0.77), respectively. However, as shown in Table 3, there was no correlation between the DBIL, ALT, or AST levels and MS.

\section{Correlation analysis of serum bilirubin and transaminase} levels and MS at baseline with different components

In the populations with zero or one MS component at baseline, multivariate Cox regression analysis showed that the parameters shown in Table 4 are not related to the risk of MS. In the population with two MS components at baseline, TBIL and IBIL levels were negatively correlated with the risk of MS. Compared to the lowest quartile level (Q1), the risk HRs (95\% CI) of the TBIL Q2-Q4 group were $0.35(0.21,0.60), 0.36(0.21,0.62)$, and $0.38(0.22$, $0.64)$, respectively, and those of the IBIL Q2-Q4 group were 0.31 (0.18-0.54), 0.39 (0.23-0.66), and 0.31 (0.180.54), respectively. However, DBIL, ALT, and AST levels were not related to the risk of MS (Table 4).

\section{Discussion}

In the Xinjiang Kazakh population, we determined the incidence of MS according to the JIS diagnostic criteria and analyzed the association of serum bilirubin and transaminase levels with MS. To our knowledge, the above study may be the first study for this specific population. The cumulative incidence of MS was $36.11 \%$ $(95 \% \mathrm{CI}=0.32,0.40)$ and the incidence density was $63.10 / 1000$ person-years. Recent studies have shown that the cumulative incidence of MS in China in 7 years was $18.55 \%$ [15] and the incidence density of MS in rural areas of South Korea was 30/1000 person-years for men and 46.4/1000 person-years for women [16]. The cumulative incidence and incidence density of MS in the Xinjiang Kazakh population are significantly higher than those in the groups mentioned above. This may be related to the unique genetic background, lifestyle, and eating habits of the Kazakh people. Staple foods of the Kazakh diet include roasted naan with flour and salt, 
Table 4 Analysis of serum bilirubin, transaminase and MS at baseline with different components

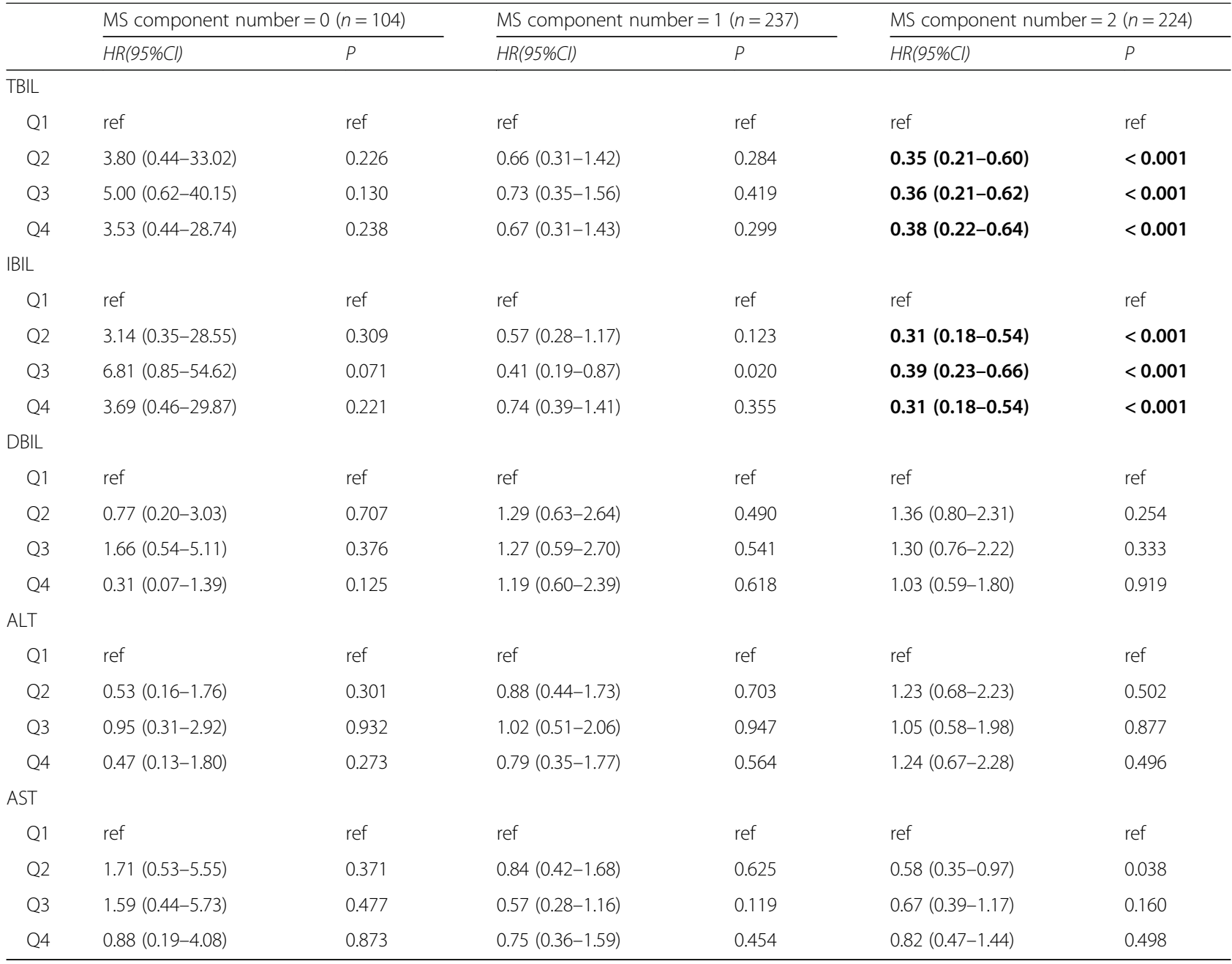

The model with adjustment for age, sex

cured meat (beef and mutton), and salty milk tea. In addition, women, especially, elderly women after marriage, rarely participate in work, field labor, and outdoor activities. The above factors lead to a higher incidence of hypertension, obesity, and dyslipidemia, which can explain the higher incidence of MS in the Kazakh population.

This study found that as the levels of TBIL and IBIL increase, the risk of MS is gradually reduced, suggesting that high levels of bilirubin have a protective effect against MS. Bilirubin is the main metabolite of heme and has long been considered a marker of liver dysfunction. Previous studies have shown that low levels of serum bilirubin increase the risk of cardiovascular diseases, diabetes, MS, obesity, and other diseases, while mildly increased serum bilirubin levels confer protection against these diseases [17]. Gilbert syndrome is a liver disorder characterized by benign hyperbilirubinemia, and studies have shown that Gilbert syndrome offers cardiovascular protection [18]. Studies have also shown that individuals with elevated bilirubin levels have a reduced risk of cardiovascular disease occurrence and death [19]. Bilirubin is recognized as the most effective endogenous antioxidant due to its continuous production in the bilirubin/biliverdin redox cycle, which can effectively scavenge hydrogen peroxide free radicals and inhibit lipid peroxidation of serum LDL [20,21]. Bilirubin is 20 times more effective than the vitamin $\mathrm{E}$ analogue Trolox in preventing LDL oxidation [22]. Other studies have found that bilirubin has an antiinflammatory effect. C-reactive protein (CRP) is a marker of chronic inflammation; several studies have shown that serum bilirubin levels are negatively correlated with CRP levels [23, 24]. Bilirubin can also inhibit the over-expression of vascular adhesion molecules induced by the inflammatory cytokine TNF- $\alpha$ [25]. In addition, mildly elevated bilirubin levels may increase insulin sensitivity. In experimental studies, bilirubin can improve the insulin sensitivity in leptin receptordeficient and diet-induced mice with obesity $[26,27]$. 
In view of the effects of bilirubin, some other studies have found a negative correlation between the total serum bilirubin levels and MS, which is consistent with the results of our study [7, 28, 29]. The results of some other studies, however, are not consistent with those of the current study. No correlation between baseline TBIL levels and MS was observed in Japanese men and women [30], and the relationship between the bilirubin subtype and MS was also inconsistent. A five-year cohort study in Chinese men showed that the levels of DBIL, but not those of TBIL and IBIL, were negatively correlated with MS [31]. This directly contradicts the results of the current study, which showed that serum levels of TBIL and IBIL, but not DBIL, were negatively correlated with MS. However, many studies have found that IBIL (also known as unconjugated bilirubin, UCB) has anti-inflammatory and anti-oxidant effects. In patients with Gilbert syndrome (increased serum UCB levels), serum bilirubin concentration was inversely related to the levels of oxidative stress markers [32]. Clinical research shows that elevated UCB levels can reduce the expression of pro-inflammatory cytokines and increase the body's antioxidant capacity [33]. In addition, evidence from experimental studies in vitro and in vivo strongly supports the data obtained in human studies. A study on the treatment of experimental colitis in a rat model showed that UCB treatment reduced the inflammatory response induced by trinitrobenzene sulfonic acid and had a strong anti-inflammatory effect [34]. Hypochlorous acid $(\mathrm{HOCl})$, an oxidant produced by myeloperoxidase (MPO), can induce protein and lipid oxidation. An in vitro experimental study showed that the addition of exogenous UCB to the serum and plasma of humans and rats can protect proteins and lipids from MPO-induced oxidation and reduce the production of chloramine and its decomposition products induced by $\mathrm{HOCl}$ and MPO [35].

Our study on the Kazakh population of Xinjiang did not show a relationship between transaminase levels and the risk of MS. This is inconsistent with the results of other studies $[6,9]$. There are two possible explanations for this: (1) the sample size of the study population is small and/or the test efficiency is low; and (2) the genetics, diets, and living habits of the study population in our study are too different from those in the previous studies.

Our study found a negative correlation between TBIL or IBIL levels and the risk of MS in a study population with two MS components at baseline. In the study population with zero or one MS component at baseline, the levels of TBIL, IBIL, DBIL, ALT, and AST were not correlated with risk of MS. This may be due to the fact that when the research subject has two components of MS, the metabolic disorder is more severe. The serum bilirubin can clear superoxide and peroxidative free radicals produced due to MS, inhibit lipid peroxidation, and fight oxidative stress, thereby enhancing the protective effects, such as reduced inflammatory response.

There are some limitations of our research. First, the small sample size of this study may limit the validity of our findings. Second, we did not measure parameters of oxidative stress, inflammatory mediators, and bilirubin metabolism-related enzymes in the study subjects. Third, each subject's bilirubin and transaminase levels were measured only once at each physical examination, which would not account for possible short-term fluctuations. Fourth, the present study did not collect information on comorbidities and medication use for data analysis.

\section{Conclusions}

This study found that serum TBIL and IBIL levels were negatively correlated with MS in the Kazakh population in Xinjiang, China; these findings may provide new options for the treatment and prevention of MS. Certain medications can be used to increase serum bilirubin levels, but there are safety concerns that will need to be addressed via further studies. Serum bilirubin levels may also be increased through nonpharmacological means, such as quitting smoking, weight loss, and the intake of the food supplement phycobilin [36, 37].

\section{Abbreviations}

MS: Metabolic syndrome; JIS: Joint Interim Statement; TBIL: Total bilirubin; IBIL: Indirect bilirubin; DBIL: Direct bilirubin; ALT: Alanine aminotransferase; AST: Aspartate aminotransferase; WC: Waist circumference; SBP: Systolic blood pressure; DBP: Diastolic blood pressure; FPG: Fasting plasma glucose; TG: Triglyceride; TC: Total cholesterol; HDL-C: High-density lipoprotein cholesterol; LDL-C: Low-density lipoprotein cholesterol; HR: Hazard ratio; 95\% Cl: 95\% confidence interval; CRP: C-reactive protein; UCB: Unconjugated bilirubin; HOCl: Hypochlorous acid; MPO: Myeloperoxidase

\section{Acknowledgments}

We sincerely thank those who participated in the study. We would also like to acknowledge the clinical laboratory of First Affiliated Hospital of Shihezi University School of Medicine for their work.

\section{Authors' contributions}

S.G. conceived and designed the study, oversaw the data analysis, and edited the manuscript. H.H. played a role in data analyses and wrote the manuscript. H.H. and H.G., and played a large role in data collection and data analysis. R.M., Y.Y., Y.H., J. M., X.Z., X.W., K.W., L.M., Y.S., J.Z., J.H., H.G., and helped collect the data. The author(s) read and approved the final manuscript.

\section{Funding}

This study was funded by the National Natural Science Foundation of China (no. 81560551) and the Shihezi University Innovation Outstanding Young Talents Program (Natural Science) (no. CXPY201908). The funders played no role in the design of this study, the collection, analysis and interpretation of data or preparation of the manuscript.

\section{Availability of data and materials}

The datasets generated during and/or analysed during the current study are not publicly available due the papers written using this dataset have not been published but are available from the corresponding author on reasonable request. 


\section{Ethics approval and consent to participate}

Before participating in the study, all subjects signed informed consent. The study was approved by the Institutional Ethics Review Board (IERB) of the First Affiliated Hospital of Shihezi University School of Medicine (IERB No. SHZ2010LL01). The study was performed in accordance with the Helsinki Declaration.

\section{Consent for publication}

Not applicable.

\section{Competing interests}

The authors declare that they have no competing interests.

\section{Author details}

'Department of Public Health, Shihezi University School of Medicine, Shihezi 832000, Xinjiang, China. ${ }^{2}$ The First Affiliated Hospital of Shihezi University Medical College, Shihezi 832000, Xinjiang, China. ${ }^{3}$ Department of Pathology and Key Laboratory of Xinjiang Endemic and Ethnic Diseases (Ministry of Education), Shihezi University School of Medicine, Shihezi 832000, Xinjiang, China.

Received: 8 March 2020 Accepted: 28 May 2020

Published online: 22 July 2020

\section{References}

1. Mendrick DL, Diehl AM, Topor LS, et al. Metabolic syndrome and associated diseases: from the bench to the clinic. Toxicol Sci. 2018;162(1):36-42.

2. Vona R, Gambardella L, Cittadini C, et al. Biomarkers of oxidative stress in metabolic syndrome and associated diseases. Oxidative Med Cell Longev. 2019;2019:8267234.

3. Mccracken E, Monaghan M, Sreenivasan S. Pathophysiology of the metabolic syndrome. Clin Dermatol. 2018;36(1):14-20

4. Cheriyath P, Gorrepati VS, Peters I, et al. High Total Bilirubin as a Protective Factor for Diabetes Mellitus: An Analysis of NHANES Data From 1999-2006. J Clin Med Res. 2010;2(5):201-6.

5. Wagner $\mathrm{KH}$, Wallner M, Molzer $\mathrm{C}$, et al. Looking to the horizon: the role of bilirubin in the development and prevention of age-related chronic diseases. Clin Sci (Lond). 2015;129(1):1-25.

6. Wang S, Zhang J, Zhu L, et al. Association between liver function and metabolic syndrome in Chinese men and women. Sci Rep. 2017;7:44844.

7. Shiraishi $\mathrm{M}$, Tanaka $\mathrm{M}, \mathrm{Okada} \mathrm{H}$, et al. Potential impact of the joint association of total bilirubin and gamma-glutamyltransferase with metabolic syndrome. Diabetol Metab Syndr. 2019;11:12

8. Schindhelm RK, Dekker JM, Nijpels G, et al. Alanine aminotransferase predicts coronary heart disease events: a 10-year follow-up of the Hoorn study. Atherosclerosis. 2007;191(2):391-6.

9. Lee SH, Cho DY, Joo NS, et al. The relationship of alanine aminotransferase to metabolic syndrome in a Korean population. Turk J Gastroenterol. 2018; 29(1):52-60

10. Pei D, Hsia TL, Chao T, et al. Gamma-glutamyl transpeptidase in men and alanine aminotransferase in women are the most suitable parameters among liver function tests for the prediction of metabolic syndrome in nonviral hepatitis and nonfatty liver in the elderly. Saudi J Gastroenterol. 2015;21(3):158-64.

11. Zhang $\mathrm{XH}_{\text {, Zhang }} \mathrm{M}, \mathrm{He}$ J, et al. Comparison of anthropometric and Atherogenic indices as screening tools of metabolic syndrome in the Kazakh adult population in Xinjiang. Int J Environ Res Public Health. 2016; 13(4):428.

12. Guo SX, Zhang XH, Zhang JY, et al. Visceral adiposity and anthropometric indicators as screening tools of metabolic syndrome among low income rural adults in Xinjiang. Sci Rep. 2016;6:36091.

13. Heng G, Rulin M, Jingyu Z, Dongsheng R, Shangzhi X, Feng S, Shuxia G. Comparison and analysis of epidemic characteristics of metabolic syndrome between Kazakh and Han ethnic groups in Xinjiang. Chin J Hypertension. 2011;19(06):538-43.

14. Alberti $\mathrm{KG}$, Eckel $\mathrm{RH}$, Grundy SM, et al. Harmonizing the metabolic syndrome: a joint interim statement of the international diabetes federation task force on epidemiology and prevention; National Heart, Lung, and Blood Institute; American Heart Association; world heart federation; international atherosclerosis society; and International Association for the Study of obesity. Circulation. 2009;120(16):1640-5.
15. Sun H, Liu Q, Wang X, et al. The longitudinal increments of serum alanine aminotransferase increased the incidence risk of metabolic syndrome: a large cohort population in China. Clin Chim Acta. 2019;488:242-7.

16. Hwang JH, Kam S, Shin JY, et al. Incidence of metabolic syndrome and relative importance of five components as a predictor of metabolic syndrome: 5-year follow-up study in Korea. J Korean Med Sci. 2013;28(12): 1768-73.

17. Vitek $L$. The role of bilirubin in diabetes, metabolic syndrome, and cardiovascular diseases. Front Pharmacol. 2012;3:55.

18. Bulmer AC, Verkade HJ, Wagner KH. Bilirubin and beyond: a review of lipid status in Gilbert's syndrome and its relevance to cardiovascular disease protection. Prog Lipid Res. 2013;52(2):193-205

19. Boon AC, Bulmer AC, Coombes JS, Fassett RG. Circulating bilirubin and defense against kidney disease and cardiovascular mortality: mechanisms contributing to protection in clinical investigations. Am J Physiol Renal Physiol. 2014;307(2):123-36.

20. Gazzin $\mathrm{S}$, Vitek L, Watchko J, et al. A novel perspective on the biology of bilirubin in health and disease. Trends Mol Med. 2016;22(9):758-68.

21. Neuzil J, Stocker R. Free and albumin-bound bilirubin are efficient coantioxidants for alpha-tocopherol, inhibiting plasma and low density lipoprotein lipid peroxidation. J Biol Chem. 1994;269(24):16712-9.

22. Wu TW, Fung KP, Yang CC. Unconjugated bilirubin inhibits the oxidation of human low density lipoprotein better than Trolox. Life Sci. 1994;54(25):477-P481.

23. Peng YF, Xie LQ, Xiang $Y$, et al. Serum bilirubin and their association with Creactive protein in patients with migraine. J Clin Lab Anal. 2016;30(6):982-5.

24. Zhao X, Li L, Li X, et al. The relationship between serum bilirubin and inflammatory bowel disease. Mediat Inflamm. 2019;2019:5256460.

25. Mazzone GL, Rigato I, Ostrow JD, et al. Bilirubin inhibits the TNFalpharelated induction of three endothelial adhesion molecules. Biochem Biophys Res Commun. 2009;386(2):338-44.

26. Takei $R$, Inoue $T$, Sonoda $N$, et al. Bilirubin reduces visceral obesity and insulin resistance by suppression of inflammatory cytokines. PLoS One. 2019; 14(10):e223302

27. Dong $H$, Huang $H$, Yun $X$, et al. Bilirubin increases insulin sensitivity in leptin-receptor deficient and diet-induced obese mice through suppression of ER stress and chronic inflammation. Endocrinology. 2014;155(3):818-28.

28. Huang SS, Chan WL, Leu HB, et al. Serum bilirubin levels predict future development of metabolic syndrome in healthy middle-aged nonsmoking men. Am J Med. 2015;128(10):1135-8.

29. Kawamoto R, Kikuchi A, Akase T, et al. Total bilirubin independently predicts incident metabolic syndrome among community-dwelling women. Diabetes Metab Syndr. 2019;13(2):1329-34.

30. Oda E, Aizawa Y. Total bilirubin is inversely associated with metabolic syndrome but not a risk factor for metabolic syndrome in Japanese men and women. Acta Diabetol. 2013;50(3):417-22.

31. Li XH, Lin HY, Guan LY, et al. Direct bilirubin levels and risk of metabolic syndrome in healthy Chinese men. Biomed Res Int. 2017;2017:9621615.

32. Maruhashi T, Soga J, Fujimura N, et al. Hyperbilirubinemia, augmentation of endothelial function, and decrease in oxidative stress in Gilbert syndrome. Circulation. 2012;126(5):598-603.

33. Sundararaghavan $V L$, Binepal $S$, Stec DE, et al. Bilirubin, a new therapeutic for kidney transplant? Transplant Rev (Orlando). 2018;32(4):234-40.

34. Zhou JA, Jiang $M$, Yang $X$, et al. Unconjugated bilirubin ameliorates the inflammation and digestive protease increase in TNBS-induced colitis. Mol Med Rep. 2017;16(2):1779-84.

35. Boon AC, Hawkins CL, Coombes JS, et al. Bilirubin scavenges chloramines and inhibits myeloperoxidase-induced protein/lipid oxidation in physiologically relevant hyperbilirubinemic serum. Free Radic Biol Med. 2015;86:259-68.

36. McCarty MF. "latrogenic Gilbert syndrome"--a strategy for reducing vascular and cancer risk by increasing plasma unconjugated bilirubin. Med Hypotheses. 2007;69(5):974-94.

37. Lin JP, Vitek L, Schwertner HA. Serum bilirubin and genes controlling bilirubin concentrations as biomarkers for cardiovascular disease. Clin Chem. 2010;56(10):1535-43.

\section{Publisher's Note}

Springer Nature remains neutral with regard to jurisdictional claims in published maps and institutional affiliations. 